\title{
ACE Inhibitors Versus ARBs Versus DRIs: A Systematic Update
}

\author{
Gerald W. Smetana, MD \\ Division of General Medicine and Primary Care, Beth Israel Deaconess Medical Center, Boston, MA, USA.
}

J Gen Intern Med 27(12):1586

DOI: $10.1007 / \mathrm{s} 11606-012-2213-3$

(c) Society of General Internal Medicine 2012

The Authors Reply-Dr. Kohlwes correctly points out that, in the treatment of hypertension, chlorthalidone is the thiazide diuretic for which we have the best evidence. Many clinicians have made a conceptual leap by assuming that hydrochlorothiazide (HCTZ) confers similar reductions in stroke, myocardial infarction, and total cardiovascular events. While intuitively plausible, this is not supported by the literature. No trials have directly compared the cardiovascular risk reduction of the two diuretics head-to-head. However, in a recent meta-analysis of nine randomized trials that included a diuretic as one arm, fewer cardiovascular events occurred among patients receiving chlorthalidone than among those who received HCTZ. ${ }^{1}$ In addition, in the MRFIT trial, fewer cardiovascular events occurred for patients taking chlorthalidone than for those taking $\mathrm{HCTZ}^{2}$; however, the choice of diuretic was not randomly assigned. An important difference that may account for these observations is the longer half-life of chlorthalidone, which confers better reduction in nighttime blood pressure than HCTZ. ${ }^{3}$ These data provide another example of the observation that all drugs that lower blood pressure do not equally reduce cardiovascular risk. ${ }^{4}$

Several practical barriers exist. Chlorthalidone is approximately twice as potent as HCTZ. Therefore, the starting dose should be $12.5 \mathrm{mg}$ daily; however, only $25 \mathrm{mg}$ and $50 \mathrm{mg}$ tabs are available. Patients must split the pills. Not all retail pharmacies routinely stock chlorthalidone. Combination antihypertensive medications simplify treatment regimens and improve adherence. However, at present, the only combination antihypertensive medications that include chlorthalidone are those with a beta blocker, clonidine, or an angiotensin receptor blocker. None of these are optimal first-line dual therapies for the treatment of hypertension. ${ }^{4}$ No combination medications exist that include ACE inhibitors or calcium channel blockers. In addition, chlorthalidone probably causes more hypokalemia than HCTZ; it is particularly important to measure serum electrolytes 2-3 weeks after starting the drug.

Once clinicians begin to prescribe chlorthalidone more often, we should expect the pharmaceutical industry to respond with lower dose tablets and more combination pills to improve adherence. For new starts, clinicians should prescribe chlorthalidone when a diuretic is indicated for the treatment of hypertension. For patients who have been stable on HCTZ, we should discuss the more robust evidence supporting chlorthalidone with our patients, and consider changing HCTZ to chlorthalidone.

Corresponding Author: Gerald W. Smetana, MD; Division of General Medicine and Primary Care, Beth Israel Deaconess Medical Center, 330 Brookline Ave., Boston, MA 02215, USA (e-mail: gsmetana@bidmc.harvard.edu).

\section{REFERENCES}

1. Rousch GC, Holford TR, Guddati AK. Chlorthalidone compared with hydrochlorothiazide in reducing cardiovascular events: systematic review and network meta-analyses. Hypertension. 2012;59:1110-1117.

2. Dorsch MP, Gillespie BW, Erickson SR, et al. Chlorthalidone reduces cardiovascular events compared with hydrochlorothiazide: a retrospective cohort analysis. Hypertension. 2011;57:689-694.

3. Sica DA. Chlorthalidone. Has it always been the best thiazide-type diuretic? Hypertension. 2006;47:321-322.

4. Smetana GW. Newer is not always better. All antihypertensive medications do not equally reduce cardiovascular risk. J Gen Intern Med. 2012;27:618-620.

Published online September 11, 2012 